\title{
Intelligent control system of tea garden lamps based on ZigBee
}

\author{
Zhanzheng $\mathrm{Xu}^{1}$, Yingming $\mathrm{Gao}^{2}$, Yidi $\mathrm{Chen}^{3}$, Huiyang Feng ${ }^{4}$, Guanying Cao* \\ \{xuzhanzheng@163.com,gycao@dlpu.edu.cn\} \\ Research Institute of Photonics, Dalian Polytechnic University Dalian 116034, China
}

\begin{abstract}
An intelligent control system for tea garden lamps is designed based on ZigBee technology. Image sensors, photosensitive sensors, temperature and humidity sensors located at the ZigBee terminal nodes are applied to monitor the tea garden environment. Real-time information of the tea garden is obtained and transported to the intelligent terminal by wireless communication network as feedback. The intelligent terminal releases instructions to control the working state of the pest phototactic lamps located at the terminal nodes which induces the pests in the tea garden to be killed by the frequency-vibration insecticidal lamps. According to the wavebands that promotes the photosynthesis of tea, a tea growth lamp is designed to solve the lack of light encountered during the growth of tea. The systematic design can not only effectively control pests, improve tea quality and yield of tea, but also reduce the use of chemical reagents and protect the natural environment.
\end{abstract}

Keywords: ZigBee technology, intelligent control, tea garden lamps, sensors, phototactic lamps

\section{Introduction}

With the improvement of living standards, tea is gradually indispensable when meeting guests. At the same time, tea gardens have become an important part of the agricultural field and have developed into an eco-tourism resource. However, tea garden pests can cause great harm to tea gardens ${ }^{[1]}$, which has always plagued tea farmers. The traditional pest measures in tea gardens include insecticide board ${ }^{[2-3]}$, insecticidal lamp ${ }^{[4-6]}$ and chemical reagents. However, when the insect board encounters rainy or 
high temperature weather, the effect of the insect board will be greatly reduced. Ordinary insecticidal lamps have a single trapping type of pests, and the effect is not obvious. Using chemical reagents to kill pests will not only affect the quality of tea, but also pollute the ecological environment.

In order to pursue an intelligent and humanized life, ZigBee technology is widely used in various places. Kumar A developed an animal health monitoring system by ZigBee technology, which can acquire animal physiological information in real time ${ }^{[7]}$. Zhang Q discussed the wireless solution of greenhouse monitoring system based on ZigBee technology, and designed the wireless node, network construction and software system ${ }^{[8]}$. Khusvinder Gill uses ZigBee design to realize a home automation system, which users can access and control remotely ${ }^{[9]}$.

In order to realize the intelligent management of tea gardens, this paper designs a tea pest control system with controllable lights based on ZigBee technology. The system mainly uses monochrome LED, wireless communication technology and sensor technology to control the work of tea garden lamps to achieve the purpose of attracting different pests in different periods of time in the tea garden.And use frequency-vibration insecticidal lights to trap and kill the attracted pests. The system can not only effectively improve the management of tea gardens, but also improve the quality and yield of tea.

\section{Design of tea garden lamps}

In the process of tea growth, most tea gardens will face the dilemma of insufficient light. This dilemma will result in slow growth of tea and delayed picking. At the same time, pests such as tea loopers and tea leaf rollers will eat tea leaves, causing the appearance of the tea leaves to be incomplete, affecting the yield of tea and reducing the income of the tea garden. In view of the above problems, the purpose of promoting the growth of tea and controlling pests can be achieved by designing tea garden growth lamps and pest phototactic lamps.

\subsection{Tea growth lamps}

Wang Jiazhen et al studied that the two wavelengths of $400-520 \mathrm{~nm}$ and $610-720 \mathrm{~nm}$ contribute the most to the photosynthesis of plants ${ }^{[10]}$. Therefore, these two bands can be intercepted on the sunlight spectrum as the ideal lamp spectrum for promoting the growth of tea. And the best LED combination scheme can be obtained by using the least square method. By rationally arranging the LEDs, the light distribution is reasonable 
and the illuminance is uniform. This solution is used to supplement the light of the tea, make up for the lack of light and promote the growth of the tea. The ideal spectrum of tea garden growth lamps is shown in Figure 1.

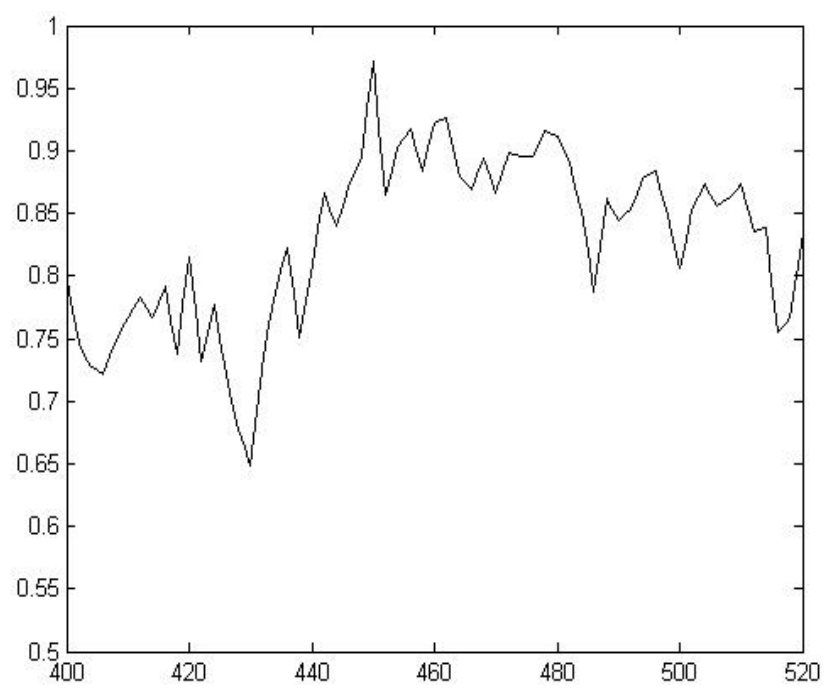

(a) blue violet spectrum

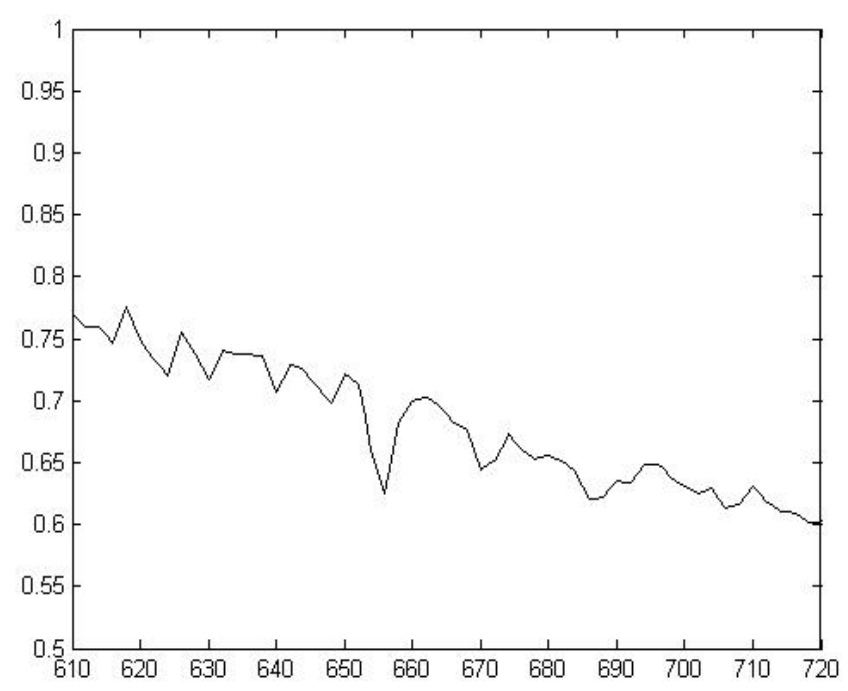

(b) red spectrum

Fig. 1. Ideal growth lamp spectrum of tea garden. 


\subsection{Phototactic lamps and insecticidal lamps}

Phototaxis is one of the most important tropisms formed by insects in the long-term evolution ${ }^{[11-12]}$. They are sensitive to the waveband of $250-700 \mathrm{~nm}$. Different types of pests in tea gardens have different light-taxis bands, as shown in Table 1.

Table 1. Phototactic band and flapping rhythm of main pests in tea garden.

\begin{tabular}{ccc}
\hline Main pest specie & Phototactic band $/ \mathrm{nm}$ & Rhythm of lighting \\
\hline Tea geometrid & $380-410$ & $19: 00-24: 00$ \\
Empoasca vitis Gothe & $410-420$ & $17: 30-19: 30$ \\
Noctuid & $330-410$ & $20: 00-24: 00$ \\
Tea leafroller & $330-400$ & $19: 00-23: 00$ \\
Aeolesthes induta Zewman & $380-450$ & $18: 00-24: 00$ \\
\hline
\end{tabular}

Taking tea geometrid as an example, tea geometrid is most sensitive to the waveband of $380-410 \mathrm{~nm}$. According to the phototactic waveband of tea geometrid, the $330-410 \mathrm{~nm}$ spectrum can be intercepted on the fitted spectrum as the ideal phototatic spectrum. By using the above method, the ideal phototaxis spectrum of other pests can be obtained, as shown in Figure 2.

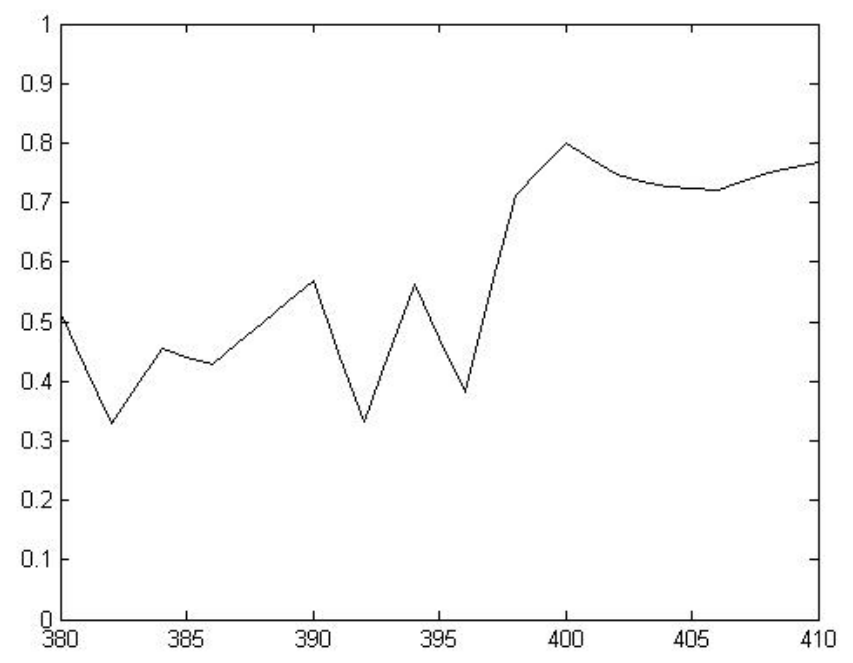

(a) phototactic spectrum of tea geometrid 


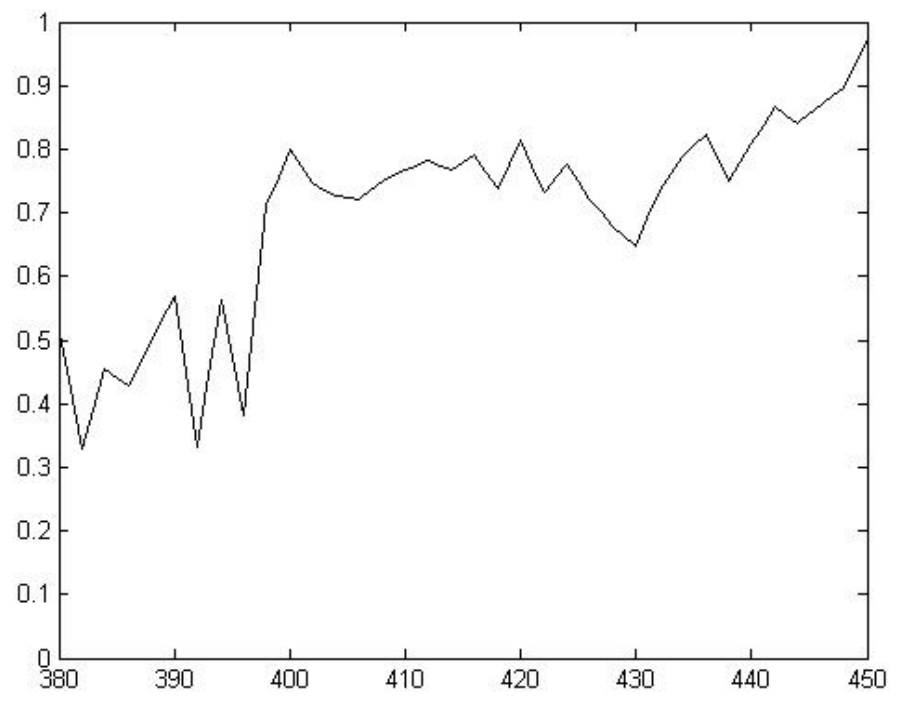

(b) phototactic spectrum of Monochamus alternatus

Fig.2. Phototactic spectrum of tea garden pests.

\section{Tea garden lamps control system}

In recent years, wireless communication technology has developed rapidly and ZigBee technology has gradually matured.The advantages of low power consumption, short time delay and high security make it more eye-catching when people choose intelligent control methods.

\subsection{System framework}

According to the difference in phototaxis of different pests at different periods and the light-fluttering rhythm ${ }^{[13-14]}$, the information is obtained and transported to the smart terminal by wireless communication network as feedback and controls the corresponding lights to work, effectively trapping pests and ensuring the normal growth of tea. The framework of the intelligent control system for tea garden lights based on ZigBee technology is shown in Figure 3. 


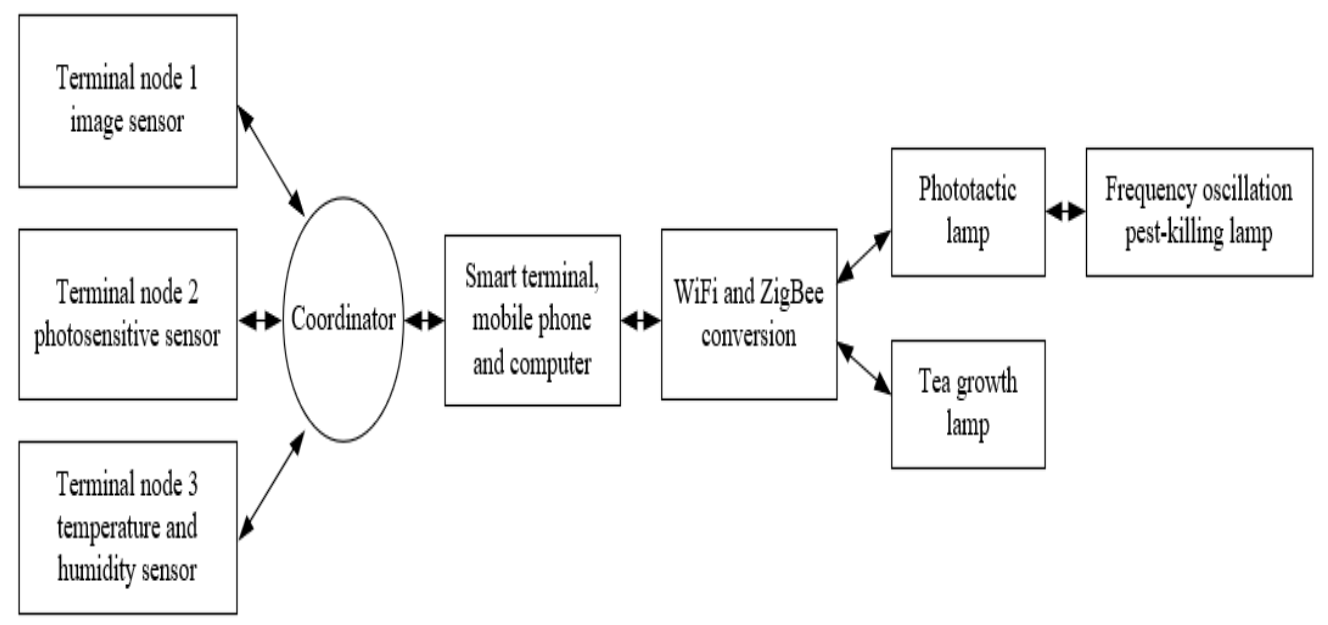

Fig.3. Framework diagram of intelligent control system for tea garden lamps.

The whole system consists of WiFi wireless local area network, ZigBee wireless personal area network, monitoring module and pest control module. The monitoring terminal node will access the preset parameters into the ZigBee LAN, and send the data measured by each sensor at the terminal node location to the coordinator in real time. The coordinator establishes a local area network according to the preset parameters and transmits the information fed back by the monitoring system to the intelligent terminal through the data interface and WiFi network. The intelligent terminal controls all kinds of phototactic lamps to work independently according to the set program instructions, and traps and kills the trapped insects through the frequency-vibrating phototactic lamp.

\subsection{Monitoring system}

The monitoring system uses sensors to monitor the environment, and sends the information to the host computer through the wireless network after processing. The monitoring module system is shown in Figure 4. 


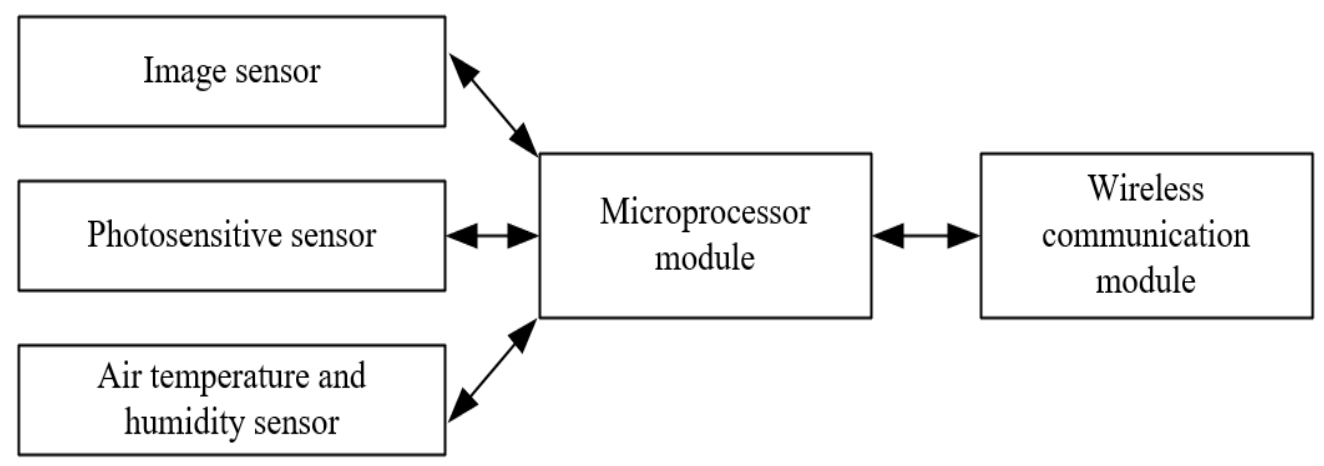

Fig.4. Monitoring module system.

(1) The image sensor is mainly used to monitor the safety of tea gardens. It is made with a 1/4 inch OV2640 million HD CMOS sensor. It has the characteristics of high sensitivity, high flexibility, supporting JPEG output and also contains data interface and control interface, which can well adapt to the different needs of the system.

(2) The photosensitive sensor is mainly used to measure light intensity information in rainy weather. When the light intensity is lower than the light compensation point suitable for the photosynthesis of tea, the managers can control the tea-growth lamp to work through the information fed back by the photosensitive sensors.

(3) The temperature and humidity sensor adopts SM51 series in the sonbest brand. This kind of sensor has low power consumption, wide measurement range and high accuracy. When the temperature and humidity are abnormal, the information can be fed back to the intelligent terminal, which is convenient for management personnel to deal with in time.

(4) The microprocessor module can mainly complete operations such as fetching instructions, executing instructions and exchanging information with external memory and logic components.

(5) The wireless communication module is responsible for establishing the network connection between each module which can realize the transmission function between the data, and has low cost and good applicability.

\subsection{WiFi and ZigBee network module}

Both WiFi and ZigBee networks are widely used wireless network communication technologies today. However, the two network protocols are not compatible. You can 
add a WiFi endpoint at the ZigBee gateway to communicate together to achieve data transmission. The WiFi local area network in this system can realize the network connection between the sensors of the monitoring system and the smart terminal (mobile phone, computer, etc.), so that the administrator can control the operation of the system through mobile phones and other devices. ZigBee network is mainly used in the field of automatic and remote control, which can realize the network connection of various lamp driving and control equipment. Reasonable use of these two wireless network communication technologies can make the management more intelligent and humanized.

\subsection{Tea garden lamps module}

In order to solve the problems of pests in tea gardens and lack of light in tea gardens, according to the light-tending band of the pests, monochromatic LEDs are used to design all kinds of pest locating lights, and then the pests are killed by frequency-vibrating insecticidal lights. The light intensity information of the tea garden is obtained and transported to the intelligent terminal by wireless communication network as feedback. When the light intensity is less than the light compensation point that promotes the photosynthesis of the tea, the system will control the light that promotes the growth of the tea to work.

In this paper, through the topological structure of the ZigBee star network, the image sensors, photosensitive sensors, temperature and humidity sensors, tea growing lights, and pest phototactic lamps are placed at the terminal nodes of the network. The information is forwarded through the coordinator to realize communication between two terminal nodes (such as photosensitive sensors and tea growth lamps).

\subsection{ZigBee network system}

In the entire ZigBee network, the node connection includes the connections between various sensors located at the the terminal nodes and the coordinator nodes. The connection between the lamps (the tea growth lamps and the pest phototactic lamps) located at the terminal nodes and the coordinator nodes. The photosensitive sensor node is successfully connected with the coordinator first, and then transmits the real-time monitoring light intensity information to the coordinator. The coordinator stores the information and compares the size with the light compensation point for photosynthesis of tea. If the light intensity measurement value is less than the light intensity value of 
the light compensation point, a command will be sent to control the operation of the tea growth lamp.The flow chart of node establishment is shown in Figure 5.

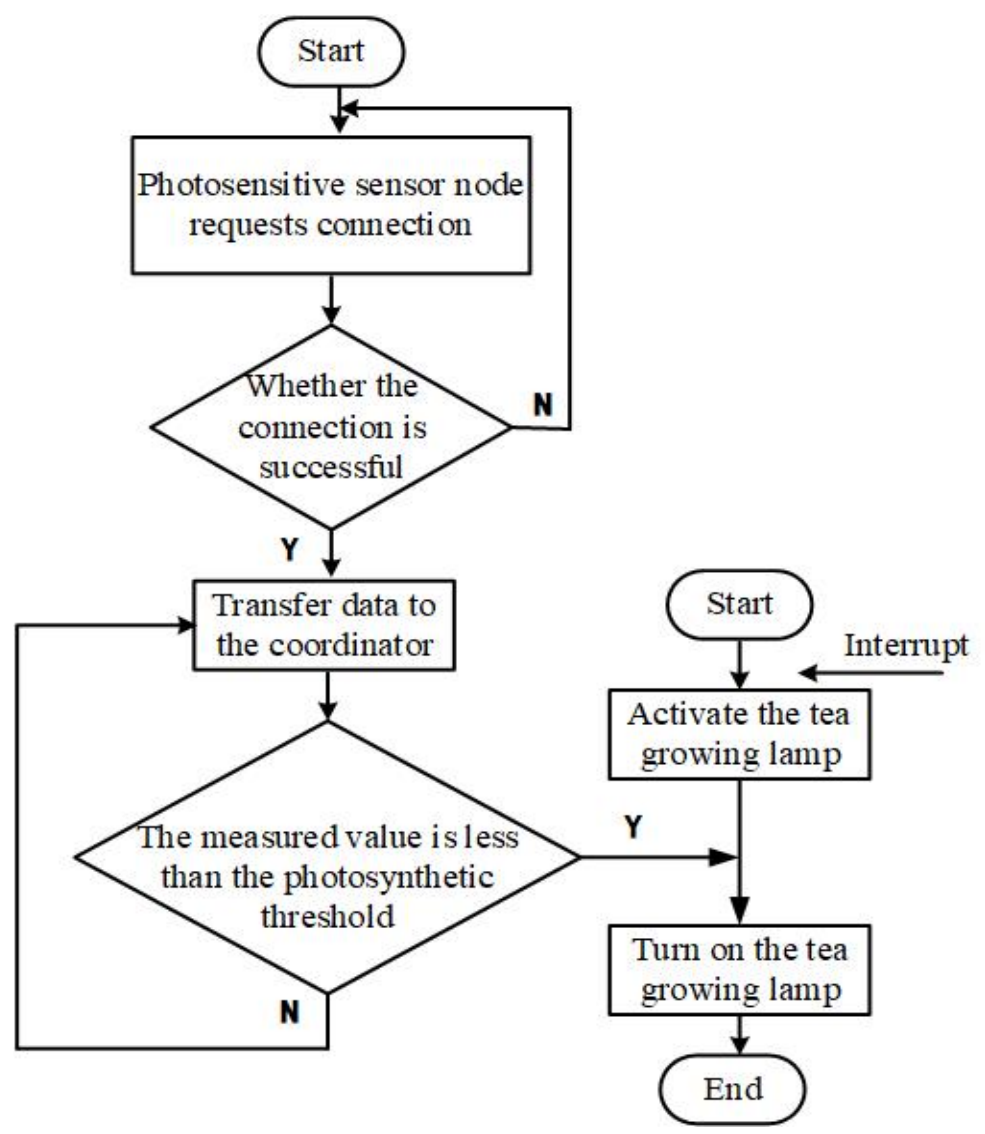

Fig.5. Terminal node establishment process.

\section{Conclusion}

The ZigBee-based pest control system for tea gardens designed in this paper, with the characteristics of ZigBee's low power consumption and short time delay which enables the system to be installed flexibly at low cost. In addition, this system uses a frequency-vibrating insecticidal lamp to catch and kill the attracted pests, which reduces the environmental pollution caused by chemical reagents. With the increasing demand for pollution-free products, the system will have a good market prospect. 


\section{References}

[1] Qi L I , Hong-Chun Z , Ji-Cai T , etc.: Occurrence and Damage of Lepidopterous-pest in Tea Gardens of Hunan[J]. Tea Communication, (2011).

[2] Hongtao Wang, Faxiang Jiang, Min Chen, etc.: Trapping effect of different color sticky insect boards on Main Pests in tea garden [J]. China plant protection guide, (2018).

[3] Yuantao Yao, Changjun Wang, Lili Tian, etc.: Study on the control effect of insect proof board on tea aphid in northern asexual tea garden [J]. Northern horticulture, (1): 112-114(2014).

[4] Li, Tianhua, Pan, et al.: Research on the LED Pest Control Light for Tea Plantations[J] Journal of Computational and Theoretical Nanoence, 13(4):2296-2299(2016).

[5] Zhaoming W, Jingfeng W, Youbing P.: The Effect of Jiaduo Multi-frequency Vibration-killing Lamps Controlling Pest in Organic Tea Garden [J]. Chinese Agricultural Science Bulletin, (2009).

[6] Xiang Xu, Li MA, Zelin Yue and other.:LED single wavelength lamp insecticidal lamps on tea garden pests $[\mathrm{J}]$. China plant protection guide, 37 (12): 53-56(2017).

[7] Kumar A, Hancke G P.: A ZigBee-based animal health monitoring system[J]. IEEE sensors Journal, 15(1): 610-617(2014).

[8] Zhang Q, Yang X, Zhou Y, etc.: A wireless solution for greenhouse monitoring and control system based on ZigBee technology[J]. Journal of Zhejiang University-Science A, 8(10): 1584-1587(2007).

[9] Gill K, Yang S H, Yao F, etc.: A ZigBee-based home automation system[J]. IEEE Transactions on Consumer Electronics, 55(2):422-430(2009).

[10] Jiazhen Wang, Xinyu Zhang, Xing Jin, etc.: Effect of night light supplement on Yield and quality of spring tea in red and blue lamp tea garden [J]. Journal of Fujian agriculture, 34 (1): 46-52(2019).

[11] Lei Bian, Xiaoling Sun, Yu Gao, etc.: Phototaxis mechanism and application progress of insects [J]. Acta Entomologica Sinica, 49 (6): 1677-1686(2012).

[12] XiangFeng J, Chaoliang L.: Advances in research on photoaxis of insects and the mechanism[J]. Kun Chong zhi shi= Kunchong Zhishi, 41(3): 198-202(2004).

[13] Haihua Tu, Xianfei Kuang, Yu Mao, etc.: Control effect of dual band led solar insecticidal lamp on tea pests [J]. China plant protection guide, 38 (10): 53-57( 2018).

[14] Haihua Tu, Xiuxia Hu, Yu Mao, etc.: Development and application of dual spectrum led insecticidal lamp for tea garden [J]. Anhui Agricultural Sciences(3): 36( 2019). 